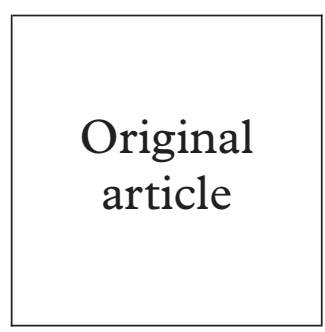

\title{
A collaborative approach to management of chlamydial infection among teenagers seeking contraceptive care in a community setting
}

\author{
N J James, S Hughes, I Ahmed-Jushuf, R C B Slack
}

Objectives: To develop and assess a coordinated model of care for effective management of genital chlamydial infection in young women, identified through a selective screening programme in a community based teenage health clinic.

Methods: Selective screening for genital Chlamydia trachomatis was undertaken among young women aged 13-19 years who were having a routine cervical smear test, being referred for termination of pregnancy, or who reported behavioural risk factors for, and/or symptoms of, genital infection. Collaboration among family planning, genitourinary medicine (GUM), and public health staff was used to enhance management of infected individuals, with particular focus on partner notification.

Results: 94 young women had confirmed genital chlamydial infection, representing $11 \%$ of those tested. All index patients received appropriate antibiotic therapy and follow up; 93 (99\%) of these were counselled by a health adviser, of whom $62(66 \%)$ were able to provide sufficient details for partner notification, resulting in treatment of male partners associated with $51(82 \%)$ of these young women. Younger age ( $\leqslant 16$ years) was significantly associated with delay in attending for treatment.

Conclusions: Effective management of genital chlamydial infection is achievable in settings outside GUM clinics using a collaborative approach which incorporates cross referencing between community based services and GUM clinics. (Sex Transm Inf 1999;75:156-161)

Keywords: chlamydia; screening; partner notification

\section{Introduction}

Recently, attention has focused on the debate concerning the need to introduce screening programmes for the control and prevention of Chlamydia trachomatis infections among sexually active women in the United Kingdom. Genital chlamydia infection rates for women attending genitourinary medicine (GUM) clinics in England and Wales peak among women aged 16-24 years, especially those aged 16-19 years. ${ }^{1}$ Similarly, recent cross sectional surveys of asymptomatic women (in the United Kingdom) attending general practices, ${ }^{2}$ and those counselled for termination of pregnancy, ${ }^{3}$ have shown young age ( $<20$ years) to be associated with the highest prevalence of chlamydial infection. This provides cause for concern since adolescent females may be at greater risk of developing sequelae than older women following chlamydial infection. ${ }^{4}$ However, it has been estimated that only $10 \%$ of genital chlamydial infections in the United Kingdom are identified in GUM clinics, while the majority of such infections are likely to be asymptomatic and may be broadly distributed among young people within the general population, particularly women aged 16-19 years. ${ }^{5}$ This highlights the need for efforts to target young sexually active women for selective screening for $C$ trachomatis in a range of different settings.

Partner notification (also referred to as contact tracing) forms an integral part of the prevention, management, and control of sexually transmitted diseases, and is particularly impor- tant in relation to $C$ trachomatis because of the high proportion of asymptomatic cases. ${ }^{5}$ The aim of partner notification is to reduce the burden of asymptomatic disease in the community and shorten the average period of infectiousness for a given disease, with a view to a reduction of disease transmission within the population. ${ }^{67}$ Traditionally, patients attending GUM clinics have been the initial focus for such efforts, although partner notification for chlamydia has, in general, not been afforded the same priority as gonorrhoea, ${ }^{8}$ and there are no national guidelines available as there are for syphilis and gonorrhoea. ${ }^{4}$ Where partner notification has been undertaken for cases of chlamydia diagnosed in community settings in the United Kingdom, this has achieved limited success. ${ }^{9}{ }^{10}$

There is clearly a need for selective screening programmes for chlamydia to include partner notification for effective management of infected individuals. Such a strategy has been identified as an essential component of a new UK pilot programme for chlamydia screening in community based settings. ${ }^{11} 12$ There have, however, been few published reports which have examined the feasibility of partner notification and its impact on health services in settings outside GUM clinics. ${ }^{4}$ This paper describes a model of care for effective management of chlamydial infection, detected through selective screening and based upon collaboration between staff working in a community family planning clinic for teenagers (the "teen- 
age" clinic), the department of genitourinary medicine, the Public Health Laboratory Service (PHLS), and the school nursing service.

\section{Methods}

Selective screening for chlamydial infection was undertaken between June 1995 and June 1997, among females aged 13-19 years who were attending a city centre teenage clinic for contraceptive care. ${ }^{13}$ Before introduction of the screening programme, an education and training session about genital chlamydial infection was provided for all family planning staff by the department of genitourinary medicine. Screening criteria were defined in a protocol as follows: all females aged 13-19 years who were either having a cervical smear, or who were being referred for termination of pregnancy (TOP) were included. Additionally, any other females within this age group who fulfilled two or more of the following criteria were offered screening for chlamydia-women who had been sexually active for at least 9 months; had engaged in unprotected sexual intercourse with a new partner within the past 3 months; had two or more different sexual partners in the past 12 months; suspected a recent sexual partner of having other sexual partners in addition to herself; or reported intermenstrual bleeding not associated with use of the progesterone only contraceptive pill and/or symptoms of genital infection. Women who had taken antibiotics in the past 3 weeks were not included in the study, and were reassessed at their next clinic visit.

Guidelines for undertaking chlamydia tests, procedures for informing women with positive test results, treatment options, and appropriate contact tracing were included in the protocol. Family planning nurses determined eligibility for screening during the routine consultation with clinic attenders, using a specifically designed registration form for an initial 3 month period, and thereafter using a screening checklist.

Young women who were eligible for screening received verbal and written information about $C$ trachomatis from a family planning nurse who then obtained written informed consent for testing and notification of positive test results to the local GUM clinic. Full explanation about the meaning of confidentiality was provided for all women, and reassurance given that confidentiality was a fundamental tenet of care provided by GUM clinics. In addition, the nurse clarified and documented a mutually agreed method of contact in the event of a positive test result. Following consultation with the nurse, all eligible women were then seen by a family planning doctor who checked that they had received verbal and written information about chlamydia, understood this information, and that consent for testing had been obtained. An endocervical swab (including the internal os) was taken according to the manufacturers' instructions and placed immediately in transport medium supplied in the test kit (IDEIA Chlamydia Specimen Collection Kit, Dako Diagnostics Ltd). All specimens were refrigerated and tested within 4 days of collection, and a fast track reporting system for test results was implemented by the local PHLS laboratory.

Direct fluorescent antibody (DFA) testing was used to confirm all positive test results. Where other genital infections were suspected from clinical examination and the sexual history of the patient, referral to the local GUM clinic was recommended by the doctor. If this was not acceptable to the patient, additional endocervical and vaginal swabs were taken at the teenage clinic to establish or exclude a diagnosis of candidiasis, trichomoniasis, and/or gonorrhoea.

Coordination and routine administration of the screening programme was undertaken by a designated family planning nurse coordinator who was also responsible for liaison between the teenage clinic and GUM clinic in relation to women with positive test results. All women who tested positive for chlamydia were immediately contacted by the clinic nurse coordinator by telephone or letter to an appropriate contact address, with an appointment to reattend the teenage clinic. In cases where no correspondence had been requested owing to concerns about confidentiality, other mutually agreed contact methods were used, including telephone calls to a named friend or contact via the school nursing service. Upon reattendance at the clinic, a doctor explained the test result and need for treatment and follow up. Women were offered treatment at the teenage clinic, or referral to the GUM clinic.

Women who chose to attend the teenage clinic for treatment were provided with appropriate antibiotic treatment by a family planning doctor (oxytetracycline $500 \mathrm{mg}$, or erythromycin $500 \mathrm{mg}$ four times a day for 7 days); where low compliance was expected or there was an urgent need for treatment before termination of pregnancy, a single dose of azithromycin (1 g) was given. An information sheet for patients about antibiotic treatment was also provided.

Counselling about the implications of chlamydial infection and the need for partner notification was provided for all females with a positive test result by a designated health adviser from the GUM clinic, who attended the teenage clinic for one session each fortnight, specifically for this purpose. During this initial discussion which included sexual health education, counselling and information about chlamydia, advice about treatment, and the need for sexual abstinence or the use of condoms, the method of partner notification was agreed.

The options were: patient referral of sexual contacts to the GUM clinic or general practitioner; provider referral undertaken by the health adviser; or conditional referral where initial attempts by the index patient were supplemented by the health adviser if no progress had been made by the follow up visit. Women were asked to return to the clinic for reassessment 1 week after completing their antibiotic treatment, and advised that if they did not attend for this appointment, the GUM clinic would be notified. Each index patient was also given advice about the risk of exposure to other 


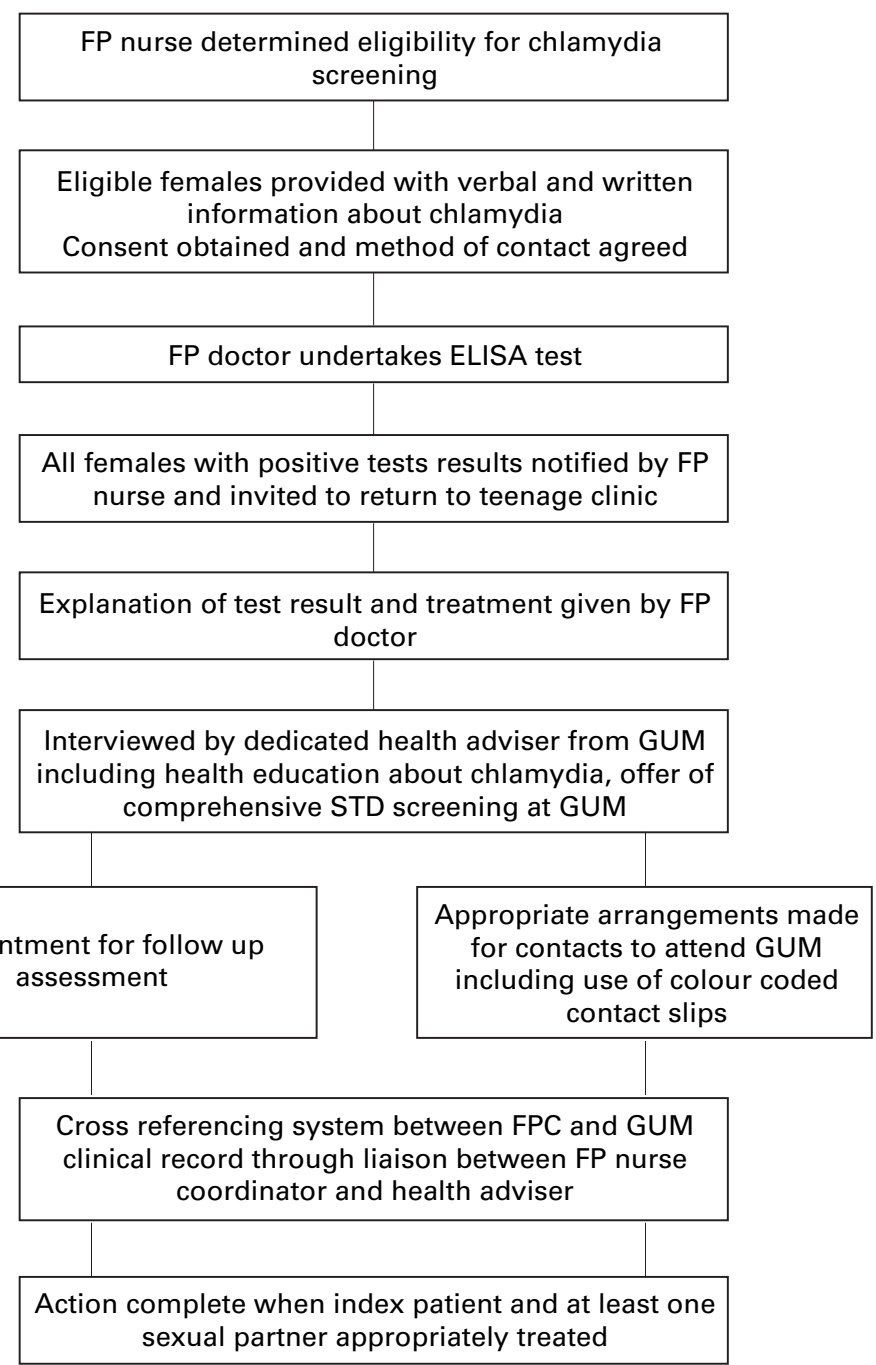

Figure 1 Patient care pathway for management of young women with chlamydial infection identified through selective screening

sexually transmitted infections (STIs) and were encouraged to take advantage of comprehensive screening at the GUM clinic; if women chose to take advantage of this service they were encouraged to attend the GUM clinic for their follow up appointment in order that screening for other STIs could be undertaken at the same time.

At the follow up appointment antibiotic compliance was checked, a family planning doctor took another sample for chlamydial testing where appropriate, and the health adviser checked what action had been taken in relation to partner notification. Where no action had been taken, consent was obtained

Table 1 Outcome of contact tracing in relation to place of treatment and follow up for females with genital chlamydial infection diagnosed in a teenage community clinic

\begin{tabular}{|c|c|c|c|c|c|}
\hline \multirow[b]{2}{*}{ Place } & \multirow{2}{*}{$\begin{array}{l}\text { Index } \\
\text { patients } \\
n(\%)\end{array}$} & \multicolumn{2}{|c|}{$\begin{array}{l}\text { Sufficient details for } \\
\text { contact tracing? }\end{array}$} & \multicolumn{2}{|c|}{$\begin{array}{l}\text { Contacts known to have } \\
\text { been treated }\end{array}$} \\
\hline & & $\begin{array}{l}\text { Yes } \\
n(\%)^{*}\end{array}$ & $\begin{array}{l}\text { No } \\
n(\%)^{*}\end{array}$ & $\begin{array}{l}\text { Yes } \\
n(\%) t\end{array}$ & $\begin{array}{l}\text { No } \\
n(\%)+\end{array}$ \\
\hline Teenage clinic & $73(77)$ & $50(68)$ & $23(32)$ & $41(82)$ & $9(18)$ \\
\hline GUM clinic & $13(14)$ & $7(54)$ & $6(46)$ & $6(86)$ & $1(14)$ \\
\hline Hospital (inpatient) & $4(4)$ & $4(100)$ & - & $3(75)$ & $1(25)$ \\
\hline Other $\ddagger$ & $4(4)$ & $1(25)$ & $3(75)$ & $1(100)$ & - \\
\hline Total & 94 & 62 & 32 & 51 & 11 \\
\hline
\end{tabular}

${ }^{\star}$ Expressed as percentage of index patients.

†Expressed as percentage of contacts for whom sufficient details were available.

‡Includes GP, other GUM clinics, and custodial care. for appropriate details to be sent to the GUM clinic with liaison provided by the designated health adviser. Action was considered complete when the following criteria were met:

(a) the patient had attended for treatment

(b) follow up with test of cure undertaken where appropriate

(c) contact tracing had been discussed and arranged by the health adviser

(d) the woman's partner(s) had been treated at the GUM clinic or elsewhere.

Frequent liaison between the clinic nurse coordinator and health adviser was necessary to monitor progress related to clinic attendance, treatment compliance, follow up, and contact tracing. The patient care pathway is summarised in figure 1 .

The outcome of contact tracing for index patients identified through the teenage clinic screening initiative was compared with that for a simple random sample of female GUM clinic attenders aged 20 years or younger and diagnosed with genital chlamydial infection between June 1995 and June 1997, excluding referrals from the teenage clinic. Interventions to contact sexual partners of index patients were undertaken in accordance with GUM clinic protocols. The number of interventions for contact tracing was therefore the same at both teenage and GUM clinics. Using information from the GUM clinic database, a list of 493 eligible female index patients was prepared, from which a random sample of 94 females was selected using computer generated random numbers. The clinic records for the sample were examined to determine the outcome of follow up and contact tracing.

\section{Results}

A total of 2667 attendances at the teenage clinic were made by females aged 13-19 for contraceptive care and advice during the study period. The total number of attendees who had an endocervical swab taken for chlamydial testing was 857 (excluding tests of cure), of whom 94 had a confirmed positive result, representing $11 \%$ (CI 8.88-13.06) of those tested. Each one of these attendees received appropriate antibiotic therapy, and all except one (subsequently serving a custodial sentence) were counselled by a health adviser at the teenage clinic following diagnosis. Each female with confirmed chlamydial infection was contacted and asked to return to the teenage clinic to discuss their test result and options for treatment. The majority, $\mathrm{n}=73(77 \%)$, chose to receive treatment and test of cure where appropriate at the teenage clinic, while $13(14 \%)$ attended the local GUM clinic, and the remaining eight $(8 \%)$ chose to attend elsewhere for treatment and follow up (see table 1).

Among the 94 index patients, 62 (66\%) provided sufficient details of their sexual partners for contact tracing, which subsequently resulted in treatment of 51 partners $(82 \%)$ associated with these women. The remaining 32 women were unable or refused to provide details of their sexual partners. Action was said to be complete for $48(51 \%)$ index patients. Examination of GUM clinic records for the 
random sample of females aged 20 years or younger was undertaken for 90 patients (four sets of records were unavailable), of whom 71 $(79 \%)$ provided sufficient details for contact tracing. This had resulted in treatment of 51 contacts $(72 \%)$, with action complete for 36 $(40 \%)$ of female index patients.

Other infections were diagnosed in addition to chlamydia for seven women (four gonorrhoea, three candida), five of whom chose the GUM clinic for treatment and test of cure, and two women with gonorrhoea who preferred treatment at the teenage clinic. Among male sexual contacts who attended the GUM clinic, the following diagnoses were made: definitive $C$ trachomatis $(\mathrm{n}=10)$, gonorrhoea $(\mathrm{n}=2)$, nongonococcal urethritis $(n=19)$, genital warts (n $=2)$, molluscum contagiosum ( $\mathrm{n}=1)$, no abnormality detected $(\mathrm{n}=17)$. Only $10 \%(\mathrm{n}=$ 9) of male contacts had urethral symptoms and all sexual contacts were treated for chlamydial infection.

Chlamydia tests were most commonly undertaken in association with cervical smears or a combination of cervical smear and other reason for testing, in accordance with the protocol (see table 2). Accordingly, the greatest proportion of positive test results was among females who had been tested for chlamydia in association with a cervical smear $(34 \%, \mathrm{n}=$ 32 ), followed by women referred for termination of pregnancy $(23 \%, \mathrm{n}=22)$, and women who reported behavioural risk factors $(15 \%, \mathrm{n}$ $=14$ ).

The mean age of females with confirmed chlamydial infection was 17.26 years (range 14-19 (SD 1.44)), of whom 40 requested no correspondence to their home address (43\%). There was no statistically significant effect of age upon the reason for testing, compliance with follow up procedures, or risk of reinfection. The number of days between the invitation to attend for treatment and actual attendance at the teenage clinic (mean $=9.8$ days, range 1-53 (SD 10.69)) was significantly greater for girls aged 16 years or less, compared with older girls $\left(\chi^{2}=9.2,3 \mathrm{df}, \mathrm{p}=0.03\right)$. Most index patients $(n=69)$ attended the clinic immediately to discuss their test result and receive treatment, although between $1-5$ recalls were necessary for the remaining 25 women (mean number of recalls 2.04 (SD 1.2)). Subsequent recalls by the nurse coordinator and/or health adviser for follow up assessment after treatment were necessary for

Table 2 Outcome of chlamydia testing in relation to reason for test

\begin{tabular}{llllll}
\hline & \multicolumn{2}{l}{ Negative result } & & \multicolumn{2}{c}{ Positive result } \\
\cline { 2 - 3 } \cline { 6 - 6 } Reason for test & $n$ & $\%$ & & $n$ & $\%$ \\
\hline Cytology & 300 & 90 & & 32 & 10 \\
TOP & 121 & 85 & & 22 & 15 \\
Risk behaviour & 142 & 91 & & 14 & 9 \\
Symptoms & 26 & 81 & & 6 & 19 \\
Multiple & 167 & 89 & & 20 & 11 \\
IUD† & 7 & 100 & & - & - \\
Total & 763 & & & 94 & 11 \\
\hline
\end{tabular}

*Two or more reasons for test (that is, combination of cytology, TOP, risk, symptoms) †Post-coital.
53 index patients, (mean number of interventions 2.25, range 1-6 (SD 1.31)). Following treatment, possible reinfection due to unprotected sex with an untreated male partner was reported by $22(23 \%)$ women.

\section{Discussion}

Surveillance data from GUM clinics indicate that young people, particularly females aged 16-19 years have the highest rates of genital chlamydial infection. ${ }^{1}$ These data may underestimate the prevalence of infection among this age group since younger sexually active people are less likely than their older counterparts to attend GUM clinics. ${ }^{14}$

At the time of study, the only available methods in Nottingham to test for chlamydia were endocervical samples examined by culture or enzyme immunosorbent assay (EIA). The latter was selected for practical reasons of storage and transport of specimens to the laboratory, as the clinic was situated some distance from the laboratory, and many specimens were taken during evening and weekend clinics. The population under study comprised young sexually active women (under 20 years), who frequently reported new sexual partners, and may therefore be expected to have a relatively high prevalence of chlamydial infection. This would tend to reduce the likelihood of false positive test results, resulting in good specificity, further improved by DFA confirmation of positive tests. However, we may expect that owing to the lower sensitivity of EIA, some false negative results were obtained. This problem is being addressed by using a polymerase chain reaction (PCR) based method in future.

In this study of attenders at a teenage family planning clinic, the overall proportion of females tested for chlamydial infection who had positive test results was $11 \%$, although this may be an underestimate of infection among this population because of practical difficulties associated with selection of women for screening on the basis of behavioural factors. Under such circumstances, special consideration must be given to the concerns of young women about a vaginal examination which includes the use of a speculum and cervical swab. ${ }^{15}$ There is therefore an urgent need to implement the use of a non-invasive screening test for chlamydia (for example, use of PCR tests for urine samples or vaginal swabs) among this population of teenagers, if they are not to be deterred from future clinic attendance for contraceptive care or, indeed, repeated screening for genital chlamydial infection.

The protocol for this study was developed before the lower age limit for cervical cytological screening was raised to age 20 in accordance with the introduction of quality standards for cervical screening in the United Kingdom ${ }^{16}$; hence, the high proportion of chlamydia tests performed in association with cervical screening. The proportion of positive chlamydia tests associated with cervical screening was, however, consistent with that reported by Grun et $a l^{2}$ for women aged under 20 attending for cervical screening/well woman checks in selected general practices in London. In common with 
other studies of chlamydial infection in the United Kingdom, ${ }^{5}$ the proportion of teenage clinic attenders with positive test results was highest among females who were selected for screening because they were seeking termination of pregnancy, or were symptomatic and would have been routinely referred to the GUM clinic.

Notification and treatment of sexual partners is a key component of the control and prevention of genital chlamydial infection. ${ }^{49}$ The small proportion of male sexual partners who had urethral symptoms in this study supports similar findings reported by Singh and Blackwell, ${ }^{17}$ who also highlighted the need for assiduous contact tracing, since the majority of male contacts are asymptomatic. There has, however, been little published research which has examined the effectiveness of patient management in settings other than GUM clinics. Recent evidence from a Danish study, ${ }^{18}$ which compared home sampling with conventional contact tracing for detecting chlamydia in male partners of infected women, showed a relatively low success rate for patient referral of sexual contacts to their general practitioners, compared with non-invasive home testing.

In the United Kingdom, current advice regarding management of patients with genital chlamydia in primary care settings recommends referral to a GUM clinic for follow up. ${ }^{19}$ However, there is some evidence to show that only a small proportion of patients diagnosed in general practice are routinely referred to a GUM clinic for follow up and contact tracing. ${ }^{20-22}$ The method and outcome of partner notification (contact tracing) and the subsequent impact on health services are therefore important considerations where screening for chlamydia is undertaken in community based settings.

The model of care developed for this study used a mutually agreed collaborative approach between family planning and GUM specialties, and resulted in the majority $(82 \%)$ of contacts for whom details were available also receiving epidemiological treatment for chlamydia. This is considerably higher than results for patient referral of male contacts to a GUM clinic by female family planning clinic attenders reported by Gunneberg et $a l,{ }^{23}$ in which $25 \%$ of male partners attended for investigation.

Since there is an increased likelihood of default from follow up at a GUM clinic associated with young people aged 25 years or less, ${ }^{24}$ a collaborative approach for the management and care may be of particular value for populations which include a high proportion of young women, as in this study. This is supported by the higher proportion of index patients at the teenage clinic for whom action was complete, compared with the randomly sampled GUM clinic comparison group. The finding that younger girls were more likely to delay than older women when attending for treatment, may be related to their concerns about confidentiality, as evidenced by the high proportion of clinic attenders who did not want any correspondence sent to their home address.
The practical implications of implementing effective cross referencing systems between community based services and GUM clinics need consideration and will require extra time and resources to ensure success. The need for good liaison between local facilities and opportunities for monitoring compliance of chlamydia positive patients referred by family planning clinics to GUM clinics has previously been recommended $^{25}$ and was an essential component of this study. We therefore suggest that the development and evaluation of interventions to control the spread of genital chlamydial infection in the United Kingdom not only include appropriate methods for partner notification but also assess the effectiveness of these activities.

We gratefully acknowledge the invaluable assistance of Anne Spells (Department of Family Planning) and Claire Brown (GUM clinic) who undertook coordination and liaison for test results and partner notification, Dr Lisa Haddon (GUM clinic) who extracted data from GUM clinic records, and Dr Simon Pugh (PHLS) for facilitating fast track reporting of test results. We also thank the patients and family planning staff at the teenage clinic, Victoria Health Centre, Nottingham. Dr Sarah Wilson (Director of Public Health) initiated and provided support for the study.

Funding: Nottingham Health Authority.

Conflict of interest: none.

Contributors: NJ designed and supervised the study, analysed the data, and wrote the paper; SH and IA provided clinical supervision and commented on drafts of the paper; RS advised on study design, organised chlamydia testing, and commented on study design, organisc
on drafts of the paper.

1 PHLS. Sexually transmitted diseases quarterly report: genital Chlamydia trachomatis infection in England and Wales. Commun Dis Rep 1997;394-5.

2 Grun L, Tassano-Smith J, Carder C, et al. Comparison of two methods of screening for genital chlamydial infection in women attending in general practice: cross sectional surin women attending in gener

3 Hopwood J, Mallinson H, Jones I. There is more to a test than technology - evaluation of testing for chlamydia infection in a charitable sector termination service. Br f Family Planning 1998;23:116-19.

4 PHLS. Genital Chlamydia trachomatis infection in England and Wales. A review of published and unpublished information with special reference to epidemiology, morbidity, screening and control. London: PHLS Communicable Disease Surveillance Centre, 1995.

5 Simms I, Catchpole M, Brugha R, et al. Epidemiology of genital Chlamydia trachomatis in England and Wales. Genitourin Med 1997;73:122-6.

6 May, RM, Anderson, RM. Transmission dynamics of HIV infection. Nature 1987;326:137-42.

7 Cowan FM, French R, Johnson AM. The role and effectiveness of partner notification in STD control: a review. Genitourin Med 1996;72:247-52.

8 McLean K. Audit of contact tracing for gonococcal and chlamydial infection. Int $\mathcal{F}$ STD AIDS 1993;4:181.

9 Johnson AM, Grun L, Haines A. Controlling genital chlamydial infection. BMF 1996;313:1160-1.

10 Boag F, Kelly F. Screening for chlamydia trachomatis (editorial). BMF 1998;316:1474.

11 Bower $\mathrm{H}$. Britain launches pilot screening programme for chlamydia. BMf 1998;316:1479.

12 Department of Health. Report of the CMO's Expert Advisory Group on Chlamydia trachomatis. London: Department of Health, Health Promotion Division, 1998.

13 Wilson S, Daniel S, Pearson J, et al. An evaluation of a new teenage clinic and its impact on teenage conceptions in teenage clinic and its impact on teenage conceptions in 86.

14 Whately J, Thin N, Reynolds B, et al. Problems of adolescent sexuality. F Roy Soc Med 1989;82:732-4.

15 Report of a Working Party. Intimate examinations. London: The Royal College of Obstetricians and Gynaecologists, 1997.

16 Quality Assurance Guidelines for the cervical screening programme. Report of a Working Party Convened by the National Health Service Cervical Screening Party (Chairman : Dr John Pritchard). Sheffield: NHSCP, 1996.

17 Singh G, Blackwell A. Morbidity in male partners of women who have chlamydial infection before termination of pregnancy. Lancet 1994;344:1438.

18 Andersen B, Ostergaard L, Moller JK, et al. Home sampling versus conventional contact tracing for detecting Chlamyversus conventional contact tracing for detecting Chlamydia trachomatis infection in male partners of
women: randomised study. BMF 1998;316:350-1.

19 Oakeshott P, Hay P. General practice update: chlamydia infection in women. Br f Gen Pract 1995;45:615-20. 
20 Owen PA, Hughes MG, Munroe JA. Study of the management of chlamydial cervicitis in general practice. $B r \mathcal{F}$ Gen Pract 1991;41:279-8

21 Mason D, Kerr S, Oakeshott P. Postal survey of managemen of cervical Chlamydia trachomatis infection in English and Welsh general practices. BMf 1996;313:1193-4

22 Ross JDC, Sutherland S, Coia J. Genital Chlamydia trachomatis infections in primary care. BMF 1996;313 1192-3
23 Gunneberg N, Mann MC, Radcliffe KW. Access to genitourinary medicine services by women attending a family planning clinic. Int $\mathcal{F}$ STD AIDS 1996;7:102-5.

24 Ross JDC, McIver A, Blakely A, et al. Why do patients default from follow up at a genitourinary clinic?: a multivariate analysis. Genitourin Med 1995;71:393-5.

25 Sin J, Gbolade BA, Russell A, et al. Referral compliance of chlamydia positive patients from a family planning clinic. Br F Family Planning 1996;22:155-6.

First announcement Joint Meeting of MSSVD and the ASTDA

3-7 May 2000, Baltimore Marriott Inner Harbor Hotel, Baltimore, Maryland, USA

To mark the unique nature of the millennial year, for the first time in its 78 year history the Medical Society for the Study of Venereal Diseases will hold its Spring Meeting jointly with the American Sexually Transmitted Disease Association (President, Professor Julius Schacter). Our local host will be Professor Jonathan Zenilman of Johns Hopkins Medical School. The scientific programme will consist of plenary lectures and round table discussions, delivered by world authorities. There will also be oral and poster presentations of original work.

Further mailings will follow to MSSVD and ASTDA members. People who do not belong to either of these organisations and who would like to receive further information should contact: Dr Keith Radcliffe, Honorary Assistant Secretary, MSSVD (fax: +44 (0) 121-237 5729; email: k.w.radcliffe@bham.ac.uk). 